\title{
Simplex Algorithm for Band Structure Calculation of Noncubic Symmetry Semiconductors: Application to III-nitride Binaries and Alloys
}

\author{
ENRICO GHILLINO ${ }^{\mathrm{a}}$, CARLO GARETTO $^{\mathrm{a}}$, MICHELE GOANO $^{\mathrm{a}, *}$, GIOVANNI GHIONE $^{\mathrm{a}}$, \\ ENRICO BELLOTTI ${ }^{\mathrm{b}}$ and KEVIN F. BRENNAN ${ }^{\mathrm{b}}$ \\ ${ }^{a}$ Dipartimento di Elettronica, Politecnico di Torino, corso Duca degli Abruzzi 24, I-10129 Torino, Italy; \\ ${ }^{\mathrm{b}}$ Microelectronics Research Center, Georgia Institute of Technology, 791 Atlantic Drive NW, \\ Atlanta, GA 30332-0269, USA
}

\begin{abstract}
A set of software tools for the determination of the band structure of zinc-blende, wurtzite, $4 \mathrm{H}$, and $6 \mathrm{H}$ semiconductors is presented. A state of the art implementation of the nonlocal empirical pseudopotential method has been coupled with a robust simplex algorithm for the optimization of the adjustable parameters of the model potentials. This computational core has been integrated with an array of Matlab functions, providing interactive functionalities for defining the initial guess of the atomic pseudopotentials, checking the convergence of the optimization process, plotting the resulting band structure, and computing detailed information about any local minimum. The results obtained for wurtzite-phase III-nitrides ( $\mathrm{AlN}, \mathrm{GaN}, \mathrm{InN})$ are presented as a relevant case study.
\end{abstract}

Keywords: Gallium nitride and related compounds; Band structure calculation; Empirical pseudopotential method; Virtual crystal approximation; Optimization methods

\section{INTRODUCTION}

The simulation of transport and optical properties of semiconductors is based on the availability of an accurate description of the band structures for the materials under investigation. Ab initio techniques [1] have become the standard methods for predicting the electronic band structure, and provide invaluable physical insight particularly in the absence of reliable experimental information. In spite of the predominance of $a b$ initio methods, the Empirical Pseudopotential Method (EPM) [2] remains the method of choice for full-band transport studies [3]. Even considering its limitations, EPM provides an optimum compromise between complexity, accuracy, and computational efficiency. It is then important to realize that, in order to provide reliable transport data, the band structures obtained with EPM have to be of $a b$ initio quality. The optimization of EPM band structures to this goal is not a trivial task, especially for novel semiconductor materials with

*Corresponding author. e-mail: goano@polito.it 
complex crystal structures. As opposed to relatively simple cubic materials, where only a few band features need to be considered, in non-cubic materials several different band details have to be optimized at the same time. Besides the (anisotropic) band edge effective masses, the energy gaps at all the symmetry points of the Brillouin zone (BZ), and the band features along the symmetry lines have to be reproduced correctly. Of particular importance are the symmetry of the pseudowavefunctions and the modifications of the electronic structure as a result of external perturbations, such as deformation potentials.

To this end, we have developed a set of integrated, interactive software tools which may greatly help in the process of optimizing the best EPM band structure of a semiconductor compound.

At the core of our computational method is the implementation of a nonlocal empirical pseudopotential code, including relativistic corrections, for zinc-blende, wurtzite, $4 \mathrm{H}$ and $6 \mathrm{H}$ type crystals. A robust simplex algorithm allows the optimization of the EPM adjustable parameters in order to match a set of reference values, i.e., energy gaps in a user-defined set of points of the BZ, and effective masses along all the principal directions, both for direct and indirect-gap materials. Particular attention has been devoted to the selection of an appropriate parametrization for the effective atomic potentials.

This computational procedure has been integrated with an array of visualization tools, providing interactive functionalities for defining the local part of the effective atomic pseudopotentials, checking the convergence of the optimization process, plotting the resulting band structure, and computing detailed information about e.g., the effective masses and nonparabolicity coefficients of the relevant valleys, and the position and value of minima not located at symmetry points.

As a relevant case study we have chosen the important family of wurtzite III-nitrides $[4,5]$. We have determined an optimized form of the local and nonlocal contributions to the effective atomic potentials of AlN, GaN, and InN. The corresponding band structures are in very good agreement with the available experimental and $a b$ initio values [6]. The model has been applied also to the important ternary alloys $\mathrm{AlGaN}$ and InGaN in the framework of the virtual crystal approximation [7].

\section{MODEL AND OPTIMIZATION METHODOLOGY}

The aim of the empirical pseudopotential method, as opposed to $a b$ initio approaches, is to build directly the effective atomic potentials starting from the knowledge of some band structure features. Historically, only a few samples of the effective potentials at some reciprocal lattice vectors (the form factors) were determined [2]. In this situation the gross features of the band structure could be obtained by adjusting the form factor values, often neglecting their physical meaning.

The first important feature of the present methodology is the implementation of physically reasonable effective potentials for the atomic components. For the local part, empirical models from the literature and results from $a b$ initio methods have been benchmarked, and a new model has been proposed [6]. In our model, each feature of the atomic potential is controlled independently by a different adjustable parameter, allowing an easier definition of the constraints on the allowed values of the parameters and an overall better conditioning of the optimization process than with other parametrizations (see e.g. $[8,9])$. The nonlocal part has been implemented using standard square potential wells with an adjustable radius and energy depth. Optionally the spin-orbit correction can be introduced as well.

The parameter set defining the EPM potentials is selected by iteratively examining how variations in the parameters alter the agreement between selected quantities extracted from the EPM calculated band structure and target values obtained from experimental results or ab initio 
calculations. For each semiconductor material, we define the functional:

$$
F=\sum_{i=1}^{n} \frac{\mid f_{i}\left(\mathrm{EPM}-\left.f_{i}(\text { target })\right|^{w_{i}}\right.}{\Delta_{i}}
$$

where $\left\{f_{\mathrm{i}}(\right.$ target $\left.)\right\}$ is a set of reference values that include the energy of the first conduction band at all high symmetry points $(\Gamma, \mathrm{M}, \mathrm{K}, \mathrm{A}, \mathrm{L}, \mathrm{H})$, the energy of several conduction bands at $\Gamma$, the total width of the valence band, the correct degeneracy and crystal field splitting among the top three valence bands, and the effective masses at $\Gamma$ for the first conduction band and the top three valence bands. The set $\left\{f_{\mathrm{i}}(\mathrm{EPM})\right\}$ collects the corresponding values generated by EPM with a given choice of adjustable parameters; the tolerances $\Delta_{i}$ are related to the maximum difference allowed between $f_{\mathrm{i}}(\mathrm{EPM})$ and $f_{\mathrm{i}}$ (target), and the exponents $w_{\mathrm{i}}$ allow a finer weighting of the various targets and a faster overall convergence.

Nonlocal pseudopotential optimization in complex semiconductors, such as wurtzite, $4 \mathrm{H}$ and $6 \mathrm{H}$ crystals is a critical process. Several optimization algorithms have been tested in order to perform the search of a (possibly global) minimum of the highly nonlinear functional (1). Gradient-based methods, Powell's method, and accelerated grid search [10] are hampered by the presence of many secondary minima, and brute force random search ("Monte Carlo", used e.g., in $[11,12]$ ) is too inefficient because of the fairly high number of constraints and adjustable parameters. Our optimization process is based on a very robust, simplex-like algorithm [13] and on a small set of operating rules:

(1) when starting from a raw initial guess, during the first iterations the local potential of each atom in the compound is optimized separately;

(2) the weight of the targets is changed during the optimization: at the begining, only a few fundamental constraints are taken into account (energy gap at $\Gamma$, valence band degeneracy, and crystal field splitting); as the process converges, the other targets are included iteratively into the functional.

(3) the nonlocal correction is included only after the local potential satisfies the fundamental constraints on the energy gaps; the inclusion of nonlocal contributions allows significantly better agreement with experimental/ab initio results, especially for the total width of the valence bands and the electron effective masses.

The complexity introduced by the necessity to search an $n$-dimensional parameter space requires the selection of an initial guess for the search that should be as close as possible to the final results. This is clearly a very difficult goal to achieve. To this end a set of preprocessing tools has been developed in order to analyze the preliminary input data.

The initial guess for the local atomic potential can be graphically built and modified starting from data available in the literature. The tool also allows to deembed, interpolate, and average if necessary, the effective atomic potentials given from other calculations. Besides the preliminary setup, the graphical preprocessor allows the test of the sensitivity of the model potential to any adjustable parameter. This is important in order to choose the sets of weights and constraints.

Equally important is the possibility of analyzing the progress of the results of the simulation. This can become a very involved task since the band structure is essentially a four dimensional object (the three component of the wavevector plus the energy) and the usual way of plotting the band diagram may be not sufficient to evaluate its features. To this end an interactive graphical post-processor has been developed to enable the calculation of the band structure parameters at different $k$-points.

\section{A CASE STUDY: III-NITRIDES}

All of the calculations in this work have been performed using a total of 197 plane waves for 
expanding the pseudo wave functions. This value has been selected in order to have a closed set of reciprocal lattice vectors, which is essential to allow the use of symmetry transformations [14].

One of the outcomes of the optimization process in wurtzite III-nitrides has been that, no matter how the weights are set, the purely local pseudopotential $[12,15]$ cannot yield at the same time all the important features of the real band structure. This is related not only to the fact that there are less degrees of freedom, but mainly to the importance that the nonlocal correction has for this kind of materials because of e.g., the deep nitrogen potential and the presence of the $d$ orbitals of indium and gallium. It is clear from the optimization process of the local bands, that the electron effective masses can not be fit with reasonable accuracy. If masses are assigned the highest weight, the other band structure features experience unacceptable errors. Therefore, for the purely local computations, a higher priority has been given to the fitting of the energy gaps, so that the overall band structure is reasonable. With the inclusion of the nonlocal correction it is possible to obtain an excellent accuracy for all of the target features. Figure 1 compares the local and nonlocal band structures of GaN. It is evident that the local pseudopotential does not yield the correct total

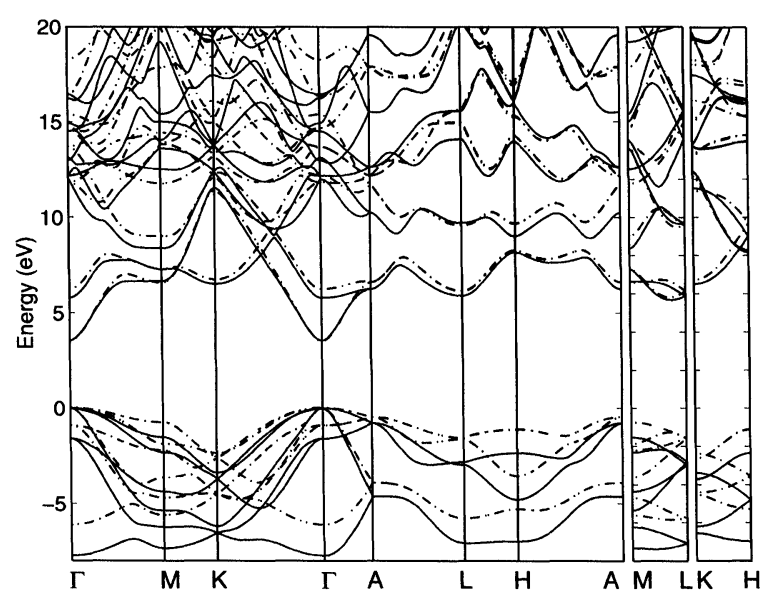

FIGURE 1 Calculated band structure of GaN. The dasheddotted line is the local result, the solid line is the nonlocal result. width of the valence band. This problem was already pointed out in [16], where the local band structure was optimized with the main target of studying high field hole transport. As opposed to the valence bands, the general features of the first two conduction bands are very close for the local and nonlocal calculations. More substantial differences are present for higher conduction bands, above $10 \mathrm{eV}$, but they do not have a great impact from the transport study standpoint.

The last feature included in our study of the III-nitrides band structure is the effect on the spin-orbit interaction, introduced as a perturbation term [17-19]. Although the spin-orbit correction is small in the nitrides, it is important for low field hole transport study. The detail of the top three valence bands of GaN including spin-orbit effect is shown in Figure 2. As an additional example of the post-processing capability the isoenergetic surfaces (at $1 \mathrm{eV}$ above the conduction band edge) for the first conduction band of $\mathrm{GaN}$ are reported in Figure 3.

Without the need of any additional parameter, the band structures of the three III-nitride ternary alloys have been computed as well with a simple VCA method. The VCA EPM values are in good agreement with the available experimental and $a b$ initio results. As a relevant example, recent

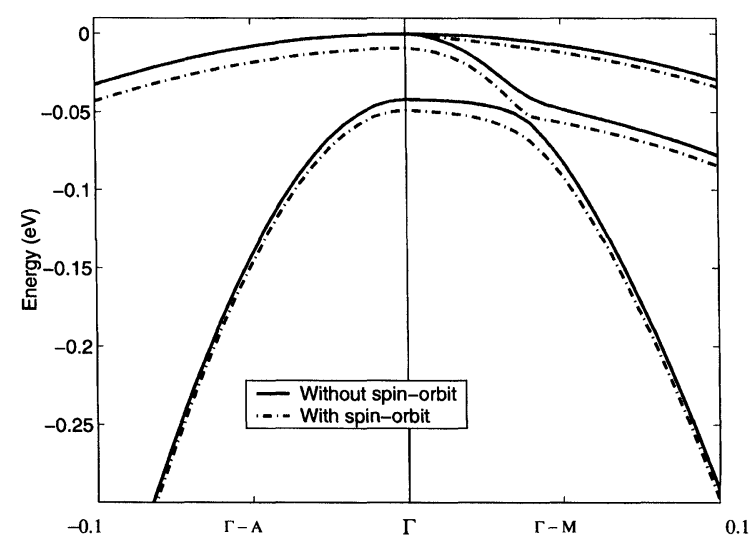

FIGURE 2 Detail of the GaN top valence bands. The solid line is the nonlocal result without spin-orbit correction, the dashed-dotted line is the case with spin-orbit correction. 


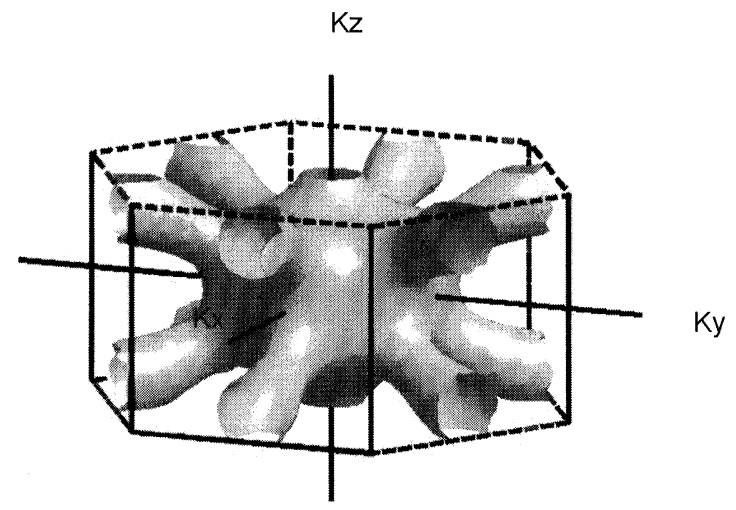

FIGURE 3 Isoenergetic surface for the first conduction band of $\mathrm{GaN}$ at $1 \mathrm{eV}$ above the band edge.

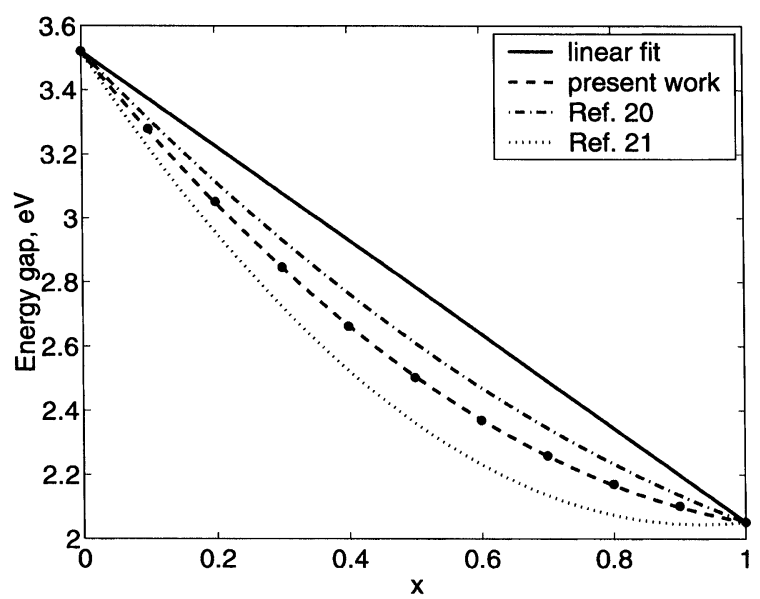

FIGURE 4 Energy gap as a function of $x$ for $\operatorname{In}_{x} \mathrm{Ga}_{1-x} \mathrm{~N}$ computed from VCA EPM, and comparison with available theoretical results.

calculations predict values of $0.7 \mathrm{eV}$ [20] and 1.7 $\pm 0.2 \mathrm{eV}$ [21] for the bowing factor of the fundamental gap in InGaN, while the present approach yields a value of $1.115 \mathrm{eV}$ (see Fig. 4).

\section{Acknowledgements}

The work at Politecnico di Torino was partially supported by CNR (National Research Council) through the MADESS II project. The work at Georgia Tech was supported in part by the National Science Foundation through grant ECS
9811366, by the Office of Naval Research through contract E21-K19, by the Office of Naval Research through subcontract E21-K69 made to Georgia Tech through the UCSB MURI program, and by the Phosphor Center of Excellence through contract E21-Z22. Usage of the Intel Corp. Advanced Platform Computer Cluster at Georgia Tech is gratefully acknowledged.

\section{References}

[1] Singh, D. J., Planewaves, Pseudopotentials and the LAPW Method, Kluwer Academic Publishers, Boston, 1994.

[2] Cohen, M. L. and Chelikowsky, J. R., Electronic Structure and Optical Properties of Semiconductors, SpringerVerlag, Berlin, 1988.

[3] Hess, K. Ed., Monte Carlo Device Simulation: Full Band and Beyond, Kluver Academic Publishers, Boston, 1991.

[4] Ambacher, O. (1998). "Growth and applications of group III-nitrides", J. Phys. D, 31, 2653-2710.

[5] Jain, S. C., Willander, M., Narayan, J. and Van Overstraeten, R., "III-nitrides Growth, characterization, and properities", J. Appl. Phys., 87(3), 965-1006, Feb., 2000.

[6] Goano, M., Bellotti, E., Ghillino, E., Ghione, G. and Brennan, K. F., "Band structure nonlocal pseudopotential calculation of the III-nitride wurtizite phase materials system. Part I: binary compounds GaN, AIN, and InN", J. Appl. Phys., 88(11), 6467-6475, Dec., 2000.

[7] Goano, M., Bellotti, E., Ghillino, E., Garetto, C., Ghione, G. and Brennan, K. F., "Band structure nonlocal pseudopotential calculation of the III-nitride wurtzite phase materials system. Part II: ternary alloys $\mathrm{Al}_{x} \mathrm{Ga}_{1-x} \mathrm{~N}$, $\mathrm{In}_{x} \mathrm{Ga}_{1-x} \mathrm{~N}$, and $\mathrm{Al}_{x} \mathrm{In}_{1-x} \mathrm{~N}^{\prime \prime}$, J. Appl. Phys., 88(11), 6476-6482, Dec., 2000.

[8] Chen, A.-B. and Srichaikul, P. (1997). "Shallow donor levels and the conduction band edge structures in polytypes of SiC", Phys. Stat. Sol. (b), 202, 81-106.

[9] Friedel, P., Hybertsen, M. S. and Schlüter, M., "Local empirical pseudopotential approach to the properties of Si/Ge superlattices", Phys. Rev. B, 39(11), 7974-7977, Apr., 1989.

[10] Basrak, Z. (1987). "A routine for parameter optimization using an accelerated grid search method", Comp. Phys. Comm., 46, 149-154.

[11] Bellotti, E., Doshi, B. K., Brennan, K. F., Albrecht, J. D. and Ruden, P. P., "Ensemble Monte Carlo study of electron transport in wurtzite InN", J. Appl. Phys., 85(2), 916-923, Jan., 1999.

[12] Pugh, S. K., Dugdale, D. J., Brand, S. and Abram, R. A. (1999). "Electronic structure calculations on nitride semiconductors", Semiconductor Sci. Tech., 14, 23-31.

[13] Gill, P. E., Murray, W. and Wright, M., Practical Optimization, Academic Press, New York, 1981.

[14] Brennan, K. F., Bellotti, E., Farahmand, M., Nilsson, H.-E., Ruden, P. P. and Zhang, Y., "Monte Carlo simulation of noncubic symmetry semiconducting materials and devices", IEEE Trans. Electron Devices, ED-47(10), 1882-1890, Oct., 2000. 
[15] Yeo, Y. C., Chong, T. C. and Li, M. F., "Electronic band structures and effective-mass parameters of wurtzite GaN and InN", J. Appl. Phys., 83(3), 1429-1436, Feb., 1998.

[16] Oğuzman, İ. H., Bellotti, E., Brennan, K. F., Kolník, J., Wang, R. and Ruden, P. P., "Theory of hole initiated impact ionization in bulk zincblende and wurtzite GaN", J. Appl. Phys., 81(12), 7827-7834, June, 1997.

[17] Weisz, G., "Band structure and Fermi surface of white tin”, Phys. Rev., 149(2), 504-518, Sept., 1966.
[18] Bloom, S. and Bergstresser, T. K. (1968). "Band structure of $\alpha$-Sn, InSb and CdTe including spin-orbit effects", Solid State Commun., 6, 465-467.

[19] Bloom, S. and Bergstresser, T. K. (1970). "Band structure of HgSe and HgTe", Phys. Stat. Sol., 42, 191-195.

[20] Kim, K., First Principles Studies of Wide Band Gap Semiconductors and Their Alloys, Ph.D. Thesis, Case Western Reserve University, Aug., 1998.

[21] van Schilfgaarde, M., Sher, A. and Chen, A.-B. (1997) "Theory of AIN, GaN, InN and their alloys", J. Cryst. Growth, 178, 8-31. 

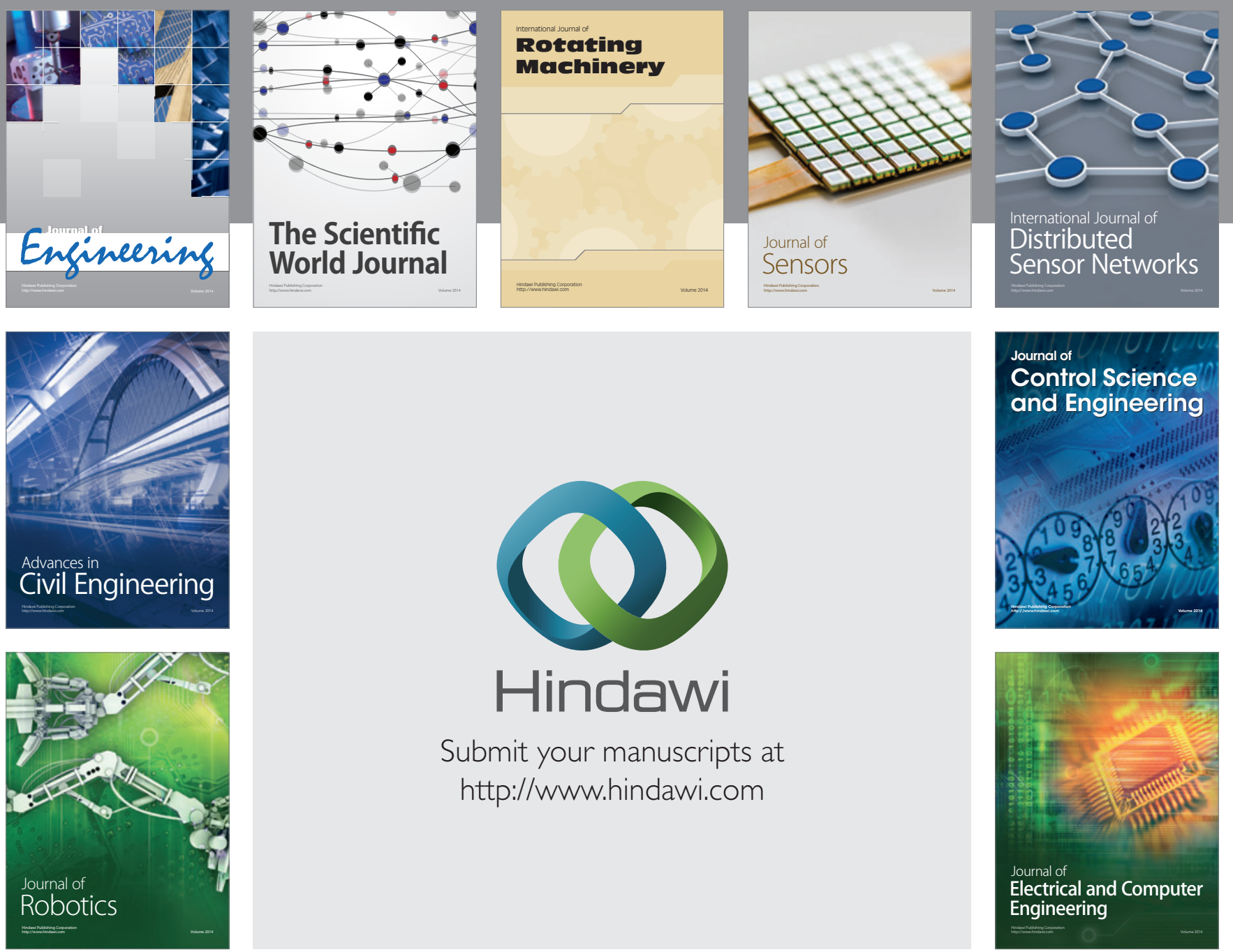

Submit your manuscripts at

http://www.hindawi.com
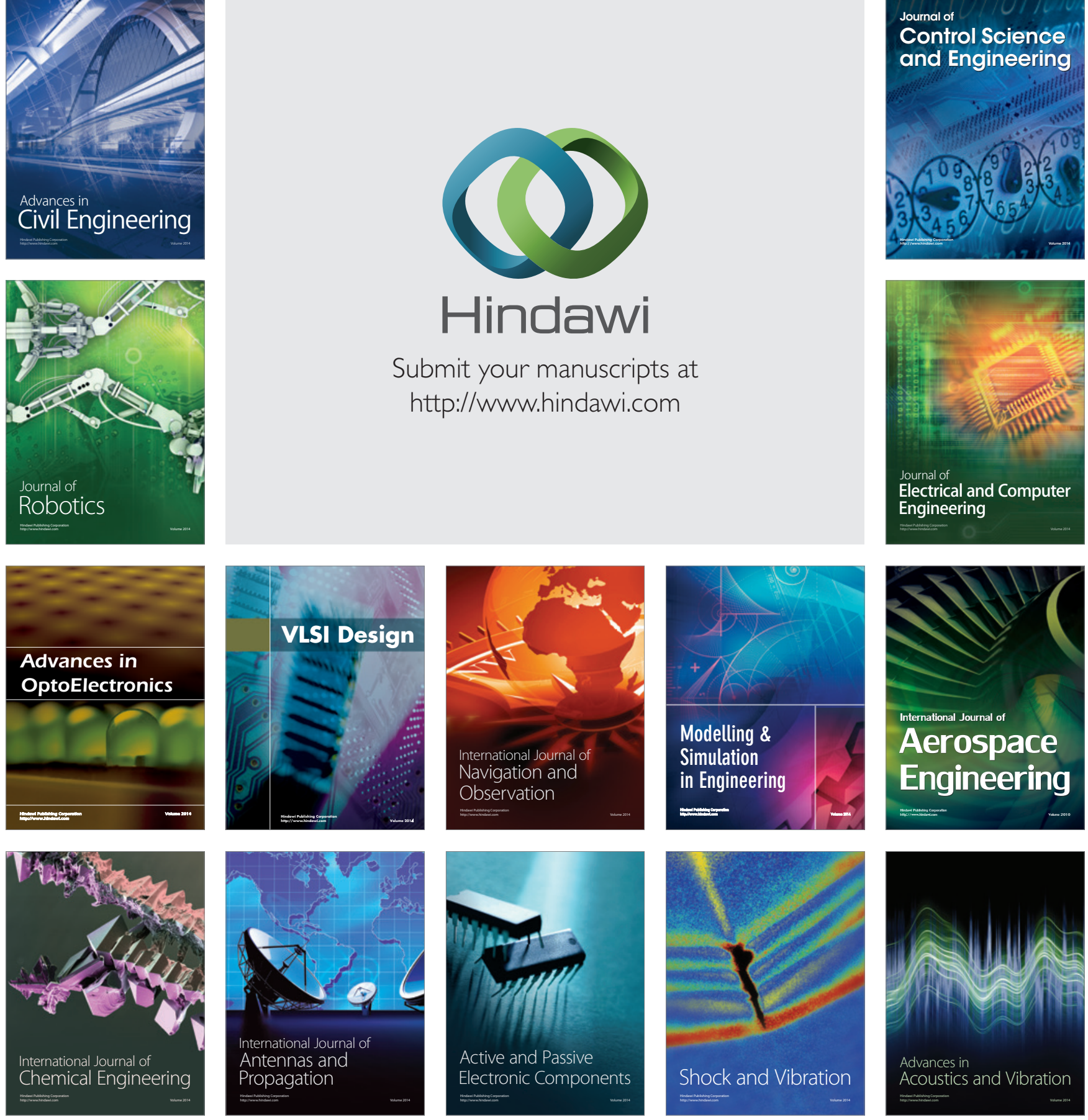